\title{
Repeat Catheter Ablation of Long-standing Persistent Atrial Fibrillation in Patients with a Total Atrial Fibrillation Duration of More Than 2 Years: Effects of the $\mathrm{CHA}_{2} \mathrm{DS}_{2}$-VASc Score and Estimated Glomerular Filtration Rate on the Outcomes
}

\author{
Qian Wang ${ }^{1,2}$, Shi-Li Jiang ${ }^{3}$, Xu Liu ${ }^{1}$ and Yi-Qing Yang ${ }^{1,2}$
}

\begin{abstract}
Objective Little is known about the outcome of repeat catheter ablation of long-standing persistent atrial fibrillation (AF) in patients with a total AF duration of more than 2 years. The main objective of this study was to explore the results and factors affecting the clinical success rate of these repeat procedures.

Methods We enrolled 99 patients with a total AF duration of more than 2 years and recurrent atrial arrhythmias after the initial catheter ablation of long-standing persistent AF. The enrolled patients were divided into two groups named the AF-recurrence group (50 patients) and the atrial tachycardia (AT)-recurrence group (49 patients) and all underwent a strict follow-up. The quality of life (QOL) and AF-related symptom classification were assessed at baseline and at 24 months post re-ablation.

Results After a mean follow-up of 31 months, 30 (30.3\%) patients were free from arrhythmia recurrence, and the success rate in the AT-recurrence group was higher than that in the AF-recurrence group $32.7 \%$ vs. $28.0 \%, \mathrm{p}=0.614$ ). A Cox regression analysis revealed a $\mathrm{CHA}_{2} \mathrm{DS}_{2}$-VASc score $\geq 3$ to be a predictor of recurrence. $\mathrm{AF}$ recurrent patients with an abnormal renal function were more prone to undergo a failed procedure. However, an abnormal renal function had no effect on the outcome of the repeat procedure for patients with AT recurrence. At the 24-month follow-up, patients maintaining sinus rhythm (SR) had a significantly improved QOL and AF-related symptoms.

Conclusion The success rate of repeat procedures for long-standing persistent $\mathrm{AF}$ and a total AF duration of more than 2 years is poor for patients with a $\mathrm{CHA}_{2} \mathrm{DS}_{2}$-VASc score $\geq 3$. An impaired renal function has an unfavorable effect on the outcome for patients with AF recurrence. For patients maintaining SR, both the QOL and AF symptomatology improve significantly.
\end{abstract}

Key words: atrial fibrillation, atrial tachycardia, repeat catheter ablation, $\mathrm{CHA}_{2} \mathrm{DS}_{2}-\mathrm{VASc}$ score, estimated glomerular filtration rate, quality of life

(Intern Med 55: 2537-2547, 2016)

(DOI: 10.2169/internalmedicine.55.5790)

\section{Introduction}

Atrial fibrillation (AF) is the most common arrhythmia found in clinical practice and it is associated with marked cardiovascular morbidity, mortality and an impaired quality of life (QOL) (1). Catheter ablation has become an established treatment method for symptomatic, recurrent, drugrefractory AF (2). Still, a great number of patients with AF require repeat ablation procedures to maximize the probabil-

\footnotetext{
${ }^{1}$ Department of Cardiology, Shanghai Chest Hospital, Shanghai Jiao Tong University, China, ${ }^{2}$ Cardiovascular Research Laboratory, and Central Laboratory, Shanghai Chest Hospital, Shanghai Jiao Tong University, China and ${ }^{3}$ Department of Internal Medicine, Laiyang Health School, China

Received for publication May 20, 2015; Accepted for publication January 17, 2016

Correspondence to Dr. Yi-Qing Yang, yiqingyang169@sina.com
} 


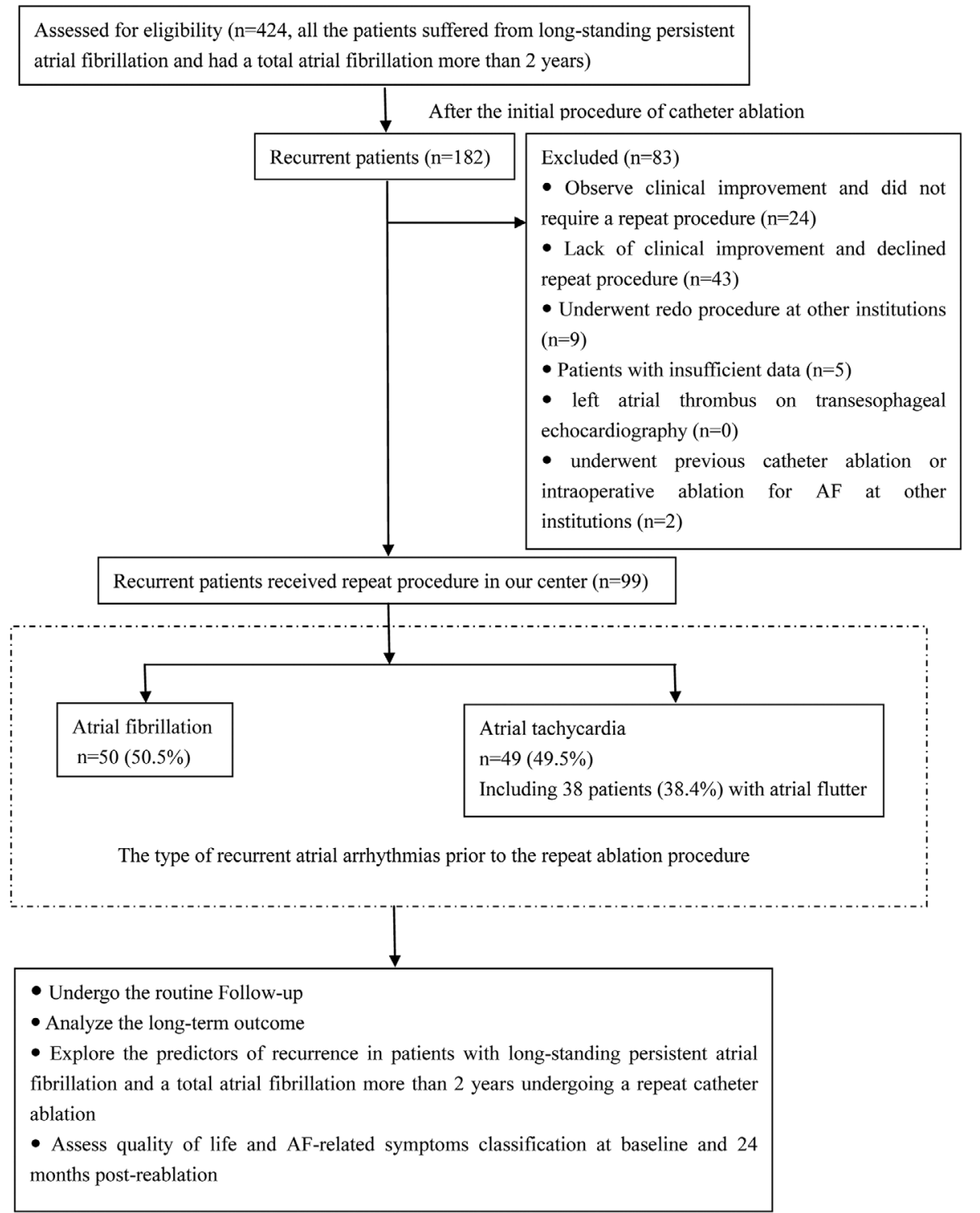

Figure 1. Study design flowchart and the type of recurrent atrial arrhythmias prior to the repeat ablation procedure.

ity of achieving a durable sinus rhythm (SR). Previous studies have reported various predictors of recurrent $\mathrm{AF}$ after the index procedure, such as the $\mathrm{CHADS}_{2}$ score, left atrial (LA) dimension (3), LA size (4), AF cycle length, AF termination (5), persistent AF duration (6), valvular heart disease, non-ischemic dilated cardiomyopathy (7) and so on. Furthermore, patients with a total AF duration of more than 2 years were almost 3 times more likely to relapse after the ablation (6). However, there is a paucity of data predicting a favorable outcome after repeat procedures for patients with long-standing persistent $\mathrm{AF}$ and a total $\mathrm{AF}$ duration of more than 2 years (8).

In the present study, we aimed to investigate the longterm outcomes after repeat procedures for patients with long-standing persistent $\mathrm{AF}$ and a total $\mathrm{AF}$ duration of more than 2 years. Moreover, we intended to respectively identify the clinical factors that could predict the redo ablation outcome for patients with $\mathrm{AF}$ recurrence and atrial tachycardia
(AT) (including atrial flutter) recurrence. Additionally, to analyze the effectiveness of catheter ablation, we evaluated the QOL and AF-related symptoms of the enrolled patients at baseline and again at 24 months post re-ablation.

\section{Materials and Methods}

\section{Participants}

A total of 424 consecutive patients with long-standing persistent $\mathrm{AF}$ and a total $\mathrm{AF}$ duration of more than 2 years undergoing the initial procedure of catheter ablation at Shanghai Chest Hospital Affiliated to Shanghai Jiao Tong University were enrolled from January 2008 to December 2011. Out of the 424 patients, 249 had recurrence after the initial procedure, and eventually 99 patients received repeat procedures in our center (Fig. 1). According to the type of recurrent atrial arrhythmia prior to the repeat procedure, we 
Table 1. Clinical Characteristics of Patients with Recurrent Atrial Arrhythmias Undergoing Redo Procedure and Those Not Undergoing Redo Procedure.

\begin{tabular}{llll}
\hline & Redo $(\mathrm{n}=99)$ & Not redo $(\mathrm{n}=76)$ & $\mathrm{p}$ Value \\
\hline Age (years) & $62.4 \pm 9.5$ & $62.6 \pm 9.6$ & 0.796 \\
Male $\mathrm{n}(\%)$ & $69(69.7 \%)$ & $48(63.2 \%)$ & 0.686 \\
Echocardiographic parameters & & & \\
$\quad$ LA dimension $(\mathrm{mm})$ & $45.0 \pm 5.0$ & $45.2 \pm 4.7$ & 0.428 \\
LVEF $(\%)$ & $60.2 \% \pm 4.9 \%$ & $60.4 \% \pm 5.5 \%$ & 0.373 \\
Comorbidities n $(\%)$ & & & \\
Hypertension & $50(50.5 \%)$ & $40(52.6 \%)$ & 0.877 \\
Diabetes mellitus & $11(11.1 \%)$ & $10(13.2 \%)$ & 0.867 \\
Coronary artery disease & $5(5.1 \%)$ & $5(6.6 \%)$ & 0.996 \\
HCM & $3(3.0 \%)$ & $2(2.6 \%)$ & 0.687 \\
DCM & $1(1.0 \%)$ & $0(0.0 \%)$ & 0.459 \\
COPD & $13(13.1 \%)$ & $10(13.2 \%)$ & 0.866 \\
Metabolic syndrome $\mathrm{n}(\%)$ & $40(40.4 \%)$ & $27(35.5 \%)$ & 0.458 \\
Body mass index $\left(\mathrm{kg} / \mathrm{m}^{2}\right)$ & $23.6 \pm 2.89$ & $22.4 \pm 3.0$ & 0.369 \\
\hline
\end{tabular}

Data are expressed as mean \pm standard deviation, or percentage

LA: left atrial, LVEF: left ventricular ejection fraction, HCM: hypertrophic cardiomyopathy, DCM: dilated cardiomyopathy, COPD: chronic obstructive pulmonary disease

divided the enrolled patients into two groups named the AFrecurrence group (50 patients) and the AT-recurrence group (49 patients). The indications for undergoing repeat procedures were: (1) an age between 18 and 80 years; (2) symptomatic and drug-refractory atrial arrhythmia after the initial procedure; (3) time since the initial procedure: a minimum of 3 months; (4) signed informed consent. Patients with insufficient data, previous catheter ablation or intraoperative ablation for AF at other institutions, left atrial thrombus on transesophageal echocardiography were excluded. The study protocol was reviewed and approved by the Institutional Ethics Committee of Shanghai Chest Hospital. Among the patients with recurrent atrial arrhythmias, there was no significant difference in the baseline characteristics between the patients undergoing repeat procedures and those not undergoing repeat procedures (Table 1).

\section{Definitions}

Procedural termination refers to atrial arrhythmias terminated and converted to SR during the repeat procedure. The first 3 months after the initial procedure were considered to be a blanking period. After the blanking period, any over30-second episode of AF and AT documented by electrocardiogram or Holter monitoring was considered to signify recurrence. $\mathrm{CHADS}_{2}$ assigns scores as follows: congestive heart failure, hypertension, age $>75$ years and diabetes mellitus count for one point each, and previous stroke or transient ischemic attack counts as two points. Compared to the $\mathrm{CHADS}_{2}$ score, $\mathrm{CHA}_{2} \mathrm{DS}_{2}$-VASc includes three additional factors: female gender, age 65-75 years, and vascular events. Each additional factor counts as one point, while an age older than 75 years was upgraded to two points. The $\mathrm{R}_{2} \mathrm{CHADS}_{2}$ score was obtained by incorporating the components of the $\mathrm{CHADS}_{2}$ score and awarding 2 points for renal dysfunction, defined as an estimated glomerular filtration rate (eGFR) $<60 \mathrm{~mL} / \mathrm{min} / 1.73 \mathrm{~m}^{2}$ (9). The four-variable Modification of Diet in Renal Disease (MDRD) study equation was used to calculate the eGFR from serum creatinine: eGFR $\left(\mathrm{mL} / \mathrm{min} / 1.73 \mathrm{~m}^{2}\right)=186.3 \times$ [serum creatinine $(\mathrm{mg} /$ $\mathrm{dL})]^{-1.154} \times$ [age (years) $]^{-0.203} \times(0.742$ if a woman $)(10)$. An abnormal renal function refers to the patients with eGFR $<90$ $\mathrm{mL} / \mathrm{min} / 1.73 \mathrm{~m}^{2}$.

\section{Pre-ablation management}

Before the scheduled procedure, all patients underwent transthoracic echocardiography and transesophageal echocardiography to evaluate the cardiac function and exclude any intracardiac thrombi. All antiarrhythmic drugs (AAD), with the exception of amiodarone, were discontinued for at least five half-lives during the periprocedural period. Patients received effective anticoagulation with warfarin for at least 1 month, with a therapeutic international normalized ratio (INR) ranging from 2.0 to 3.0. Warfarin was discontinued 3 days before the procedure, and full-dose weight-adjusted $(1.5 \mathrm{mg} / \mathrm{kg}$ daily) subcutaneous low molecular weight heparin was used to bridge the procedure until oral anticoagulation was achieved following ablation.

\section{Electrophysiological study and ablation procedure}

All 424 long-standing persistent AF patients with a total AF duration of more than 2 years followed the step-wise approach, including, in a progressive fashion, circumferential pulmonary vein isolation (CPVI), complex fractionated atrial electrograms (CFAEs) ablation followed by linear ablation as necessary to achieve the endpoint of AF termination. The methods and endpoints of ablation were described in detail in our previous study (11). During the repeat procedure, firstly, pulmonary vein (PV) isolation was reconfirmed with a circular mapping catheter at each PV, and CPVI was used to eliminate a recovery of the PV potentials. Secondly, for 
Table 2. EHRA Atrial Fibrillation Symptoms Classification.

\begin{tabular}{lll}
\hline & Symptom severity & Definition \\
\hline EHRA I & No symptoms & \\
EHRA II & Mild symptoms & Normal daily activity not affected \\
EHRA III & Severe symptoms & Normal daily activity affected \\
EHRA IV & Disabling symptoms & Normal daily activity discontinued \\
\hline
\end{tabular}

EHRA: the European Heart Rhythm Association

AF recurrence, the ablation of CFAEs was employed, and after restoring SR by ablation or cardioversion, the bidirectional blocks of the mitral isthmus (MI) line, the roof line, and the cavotricuspid isthmus (CTI) line were verified using the above-mentioned method (11). For AT, the suspected macroreentrant AT was mapped using CARTO three dimensional mapping, and ablated at the corresponding isthmus. If a focal AT was suspected, it was mapped and ablated at the site of earliest activation. After restoring SR, PV isolation and the complete bidirectional block of the MI, roof line and CTI were verified and consolidated as required.

\section{Post-ablation management}

All patients were administered low molecular weight heparin for 3-5 days after the procedure, and also took warfarin for at least 3 months, achieving the target intensity INR of 2.0-3.0. AAD (Normally, the patients take a daily oral maintenance dose of either $200-400 \mathrm{mg}$ amiodarone or $150 \mathrm{mg}$ propafenone three times a day. If the patients cannot tolerate such a dose, then we reduced the dose circumstances of each individual patient.) was administered for 3 months to prevent any early recurrence of atrial arrhythmia. After the blanking period, warfarin and AAD therapy were encouraged for patients who did not show a good response after the repeat procedure. The second redo ablation procedure was performed in the case of a recurrent drugrefractory atrial arrhythmia.

\section{Follow-up}

All patients were scheduled for regular follow-up appointments in the outpatient clinic at 1, 3, 6, and 12 months after the repeat procedure and then every 6 months. The routine investigations included transthoracic echocardiogram, 12lead surface electrocardiogram and 24-hour Holter monitoring. Monthly telephone inquiry by the referring physicians was performed to evaluate the severity of symptoms. Clinical success was defined as free from atrial arrhythmia recurrence without taking any $\mathrm{AAD}$ before the last follow-up. Furthermore, cardiac computed tomography was performed at 3 months after the procedure to evaluate the presence of PV stenosis.

\section{Quality of life assessment and EHRA atrial fibrillation-related symptoms classification}

The Medical Outcomes Study Short-Form 36 Health Survey (SF-36) and the European Heart Rhythm Association (EHRA) class were used to assess the QOL and AF-related symptoms classification at baseline (refers to the condition when the repeat procedure was performed) and at 24 months post re-ablation $(12,13)$.

All patients receiving the repeat procedures subsequently completed the SF-36 questionnaire. The SF-36 assesses health-related QOL on 8 domains pertaining to physical health (4 domains) and mental health (4 domains), namely physical functioning, role limitations due to physical health, bodily pain, general health, vitality, social functioning, role limitations due to emotional problem, and mental health, respectively. For each subscale, the scores were transformed to a scale ranging from 0 to 100 , with lower scores representing a lower QOL. EHRA assigns patients with AF into 4 EHRA classes based on symptoms and their effect on daily activity, namely no symptoms, mild symptoms, severe symptoms and disabling symptoms (Table 2) (13).

\section{Statistical analysis}

Continuous variables were expressed as the mean \pm standard deviation or median and interquartile ranges (25th and 75th percentile) when appropriate; while categorical variables were represented by frequencies and relative percentages. Between-group comparisons were performed using the t-test or Chi-square test. Univariate and multivariate predictors were identified using Cox proportional hazards regression models. Candidate variables with $\mathrm{p}$ values $<0.10 \mathrm{ac}-$ cording to univariate regression analyses were entered into a multivariate model. Relative risks were expressed as hazard ratios (HRs) with corresponding 95\% confidence intervals (CIs). The optimal cut-off value of the eGFR for predicting AF recurrence was identified using the receiver operation characteristic (ROC) curve. Event-free survival was estimated by a Kaplan-Meier survival analysis with the log-rank test. A p value $<0.05$ was considered to be statistically significant. All statistical analyses were performed using the SPSS 17.0 software program.

\section{Results}

\section{Patient characteristics}

In this single-center cohort study, after the exclusion process, we eventually included 99 patients with recurrent atrial arrhythmias and a total AF duration of more than 2 years who underwent a repeat procedure in our center. Fifty out of the 99 patients had AF recurrence. Among the remaining 49 patients, AT (including atrial flutter) was the cause of recur- 
Table 3. Baseline Characteristics of Patients Undergoing the Repeat Procedure.

\begin{tabular}{|c|c|c|c|}
\hline & AT-recurrence group $(n=49)$ & AF-recurrence group $(n=50)$ & $\mathrm{p}$ Value \\
\hline Age (years) & $63.9 \pm 9.6$ & $60.9 \pm 9.3$ & 0.128 \\
\hline Male n(\%) & $33(67.3 \%)$ & $33(66.0 \%)$ & 0.953 \\
\hline Total AF duration (years) & $5.0 \pm 3.7$ & $8.5 \pm 6.8$ & 0.022 \\
\hline Duration of persistent AF (years) & $1.7 \pm 2.0$ & $2.6 \pm 3.4$ & 0.020 \\
\hline \multicolumn{4}{|l|}{ Echocardiographic parameters } \\
\hline LA diamension (mm) & $46.2 \pm 5.3$ & $46.0 \pm 5.3$ & 0.835 \\
\hline $\operatorname{LVEF}(\%)$ & $60.7 \% \pm 4.5 \%$ & $59.7 \% \pm 5.3 \%$ & 0.167 \\
\hline \multicolumn{4}{|l|}{ Comorbidities n $(\%)$} \\
\hline Hypertension & $27(55.1 \%)$ & $23(46.0 \%)$ & 0.149 \\
\hline Diabetes mellitus & $5(10.2 \%)$ & $6(12.0 \%)$ & 0.532 \\
\hline Coronary artery disease & $3(6.1 \%)$ & $2(4.0 \%)$ & 0.226 \\
\hline Cardiomyopathy & $2(4.1 \%)$ & $2(4.0 \%)$ & 0.741 \\
\hline COPD & $5(10.2 \%)$ & $8(16.0 \%)$ & 0.703 \\
\hline Metabolic syndrome n $(\%)$ & $18(36.7 \%)$ & $22(44.0 \%)$ & 0.860 \\
\hline Body mass index $\left(\mathrm{kg} / \mathrm{m}^{2}\right)$ & $23.8 \pm 2.3$ & $23.4 \pm 2.5$ & 0.826 \\
\hline \multicolumn{4}{|l|}{ Laboratory examinations } \\
\hline LDL-cholesterol (mg/dL) & $122.9 \pm 18.4$ & $126.7 \pm 20.0$ & 0.773 \\
\hline HDL-cholesterol (mg/dL) & $54.0 \pm 9.1$ & $55.1 \pm 10.4$ & 0.687 \\
\hline Serum creatinine $(\mathrm{mg} / \mathrm{dL})$ & $0.9 \pm 0.1$ & $0.9 \pm 0.2$ & 0.068 \\
\hline eGFR $\left(\mathrm{ml} / \mathrm{min} / 1.73 \mathrm{~m}^{2}\right)$ & $88.5 \pm 19.1$ & $86.3 \pm 20.2$ & 0.524 \\
\hline Uric acid (mg/dL) & $5.4 \pm 1.6$ & $5.9 \pm 1.8$ & 0.183 \\
\hline $\mathrm{BNP}(\mathrm{pg} / \mathrm{mL})$ & $87.9 \pm 91.6$ & $174.0 \pm 173.9$ & 0.211 \\
\hline $\mathrm{CRP}(\mathrm{mg} / \mathrm{L})$ & $4.8 \pm 10.7$ & $3.4 \pm 4.4$ & 0.666 \\
\hline $\mathrm{CHADS}_{2}$ score & $1(0-1)$ & $1(0-2)$ & 0.201 \\
\hline $\mathrm{CHA}_{2} \mathrm{DS}_{2}$-VASc score & $1(0-3)$ & $2(1-3)$ & 0.095 \\
\hline $\mathrm{R}_{2} \mathrm{CHADS}_{2}$ score & $1(0-2)$ & $1(0-2)$ & 0.496 \\
\hline \multicolumn{4}{|l|}{ Medical therapy prior to the repeat procedure } \\
\hline ACEI/ARB & $22(44.9 \%)$ & $28(56.0 \%)$ & 0.746 \\
\hline$\beta$-blokers & $15(30.6 \%)$ & $11(22.0 \%)$ & 0.335 \\
\hline Statin & $10(20.4 \%)$ & $7(14.0 \%)$ & 0.334 \\
\hline Number of ineffective antiarrhythmic drugs & $2(2-3)$ & $2(2-3)$ & 0.696 \\
\hline
\end{tabular}

LDL: low density lipoprotein, HDL: high density lipoprotein, BNP: brain natriuretic peptide, CRP: C-reactive Protein.

Other abbreviations as in Table 1

rence (Fig. 1). The baseline characteristics of patients undergoing the repeat procedure are summarized in Table 3. Patients with $\mathrm{AF}$ recurrence had a longer total AF duration ( $\mathrm{p}=$ 0.022 ) and a longer duration of persistent $\mathrm{AF}(\mathrm{p}=0.020)$ than those with AT recurrence (Table 3).

\section{Antiarrhythmic drugs administration during follow- up}

After the blanking period, for patients with SR maintenance, AAD was no longer administered during follow-up. For patients who failed in the repeat procedure, AAD was firstly encouraged. After the blanking period, a total of 43 patients required ongoing $\mathrm{AAD}$ therapy for recurrent atrial arrhythmias. Amiodarone was predominantly used in 22 patients, propafenone was administered in 11 patients, and a combination of AAD therapy was used in 10 patients (amiodarone plus propafenone in 6 patients, and propafenone plus verapamil in 4 patients). Due to drug intolerance or severe side effect, 23 patients discontinued AAD administration and underwent a third procedure.

\section{Predictors of recurrence after the repeat procedure}

To analyze the predictors of recurrence after the repeat procedure, both univariate and multivariate analyses were re- spectively performed for AT recurrent patients (Table 4) and AF recurrent patients (Table 5). For patients with AT recurrence, a $\mathrm{CHA}_{2} \mathrm{DS}_{2}$-VASc score $\geq 3$ (HR 3.720, 95\% CI: 1.367-10.124, $\mathrm{p}=0.010$ ) was identified to be a significant predictor of recurrence after the repeat procedure according to a multivariate Cox proportional hazard analysis (Table 4), while for patients with $\mathrm{AF}$ recurrence, a $\mathrm{CHA}_{2} \mathrm{DS}_{2}$-VASc score $\geq 3$ (HR 2.707, 95\% CI: 1.151-6.363, p=0.022) as well as an abnormal renal function (HR 2.821, 95\% CI: $1.277-$ $6.232, \mathrm{p}=0.010$ ) was independently associated with procedural failure (Table 5).

Derived from the ROC curve, eGFR $<86 \mathrm{~mL} / \mathrm{min} / 1.73 \mathrm{~m}^{2}$ predicted recurrence significantly in the Kaplan-Meier analysis (log-rank probability value $=0.001$ ) for AF recurrent patients after the repeat procedure, with a sensitivity of $63.9 \%$ and specificity of $92.9 \%$ (area under the ROC curve $=0.812$; Fig. 2).

\section{Long-term results after the repeat procedure and corresponding arrhythmia-free survival}

Among the 99 patients who underwent the repeat procedure, 16 out of the 49 AT recurrent patients (32.7\%) were free from atrial arrhythmias; however, only 14 out of the 50 AF recurrent patients $(28.0 \%)$ remained SR during the long- 
Table 4. Cox Proportional Hazard Analysis to Recognize Variables Associated with AT Recurrence after the Repeat Procedure.

\begin{tabular}{|c|c|c|c|c|c|c|}
\hline \multirow[t]{2}{*}{ Variables } & \multicolumn{3}{|c|}{ Univariate analysis } & \multicolumn{3}{|c|}{ Multivariate analysis } \\
\hline & HR & $95 \% \mathrm{CI}$ & $\mathrm{p}$ value & $\mathrm{HR}$ & $95 \% \mathrm{CI}$ & $\mathrm{p}$ value \\
\hline Age (years) & 1.009 & $0.973-1.046$ & 0.630 & & & \\
\hline Male & 1.022 & $0.486-2.149$ & 0.955 & & & \\
\hline Total AF duration (years) $^{a}$ & 0.952 & $0.856-1.058$ & 0.360 & & & \\
\hline Duration of persistent AF (years) ${ }^{a}$ & 0.996 & $0.987-1.005$ & 0.360 & & & \\
\hline \multicolumn{7}{|l|}{ Echocardiographic parameters } \\
\hline LA diamension $(\mathrm{mm})$ & 1.061 & $0.991-1.136$ & 0.090 & 0.975 & $0.883-1.076$ & 0.975 \\
\hline LVEF (\%) & 1.061 & $0.936-1.202$ & 0.357 & & & \\
\hline \multicolumn{7}{|l|}{ Comorbidities (n) } \\
\hline Hypertension & 0.957 & $0.480-1.909$ & 0.900 & & & \\
\hline Diabetes mellitus & 1.428 & $0.501-4.068$ & 0.505 & & & \\
\hline $\mathrm{HCM}$ & 1.333 & $0.181-9.849$ & 0.778 & & & \\
\hline DCM & 1.825 & $0.245-13.587$ & 0.557 & & & \\
\hline COPD & 0.914 & $0.739-1.133$ & 0.413 & & & \\
\hline $\mathrm{CHADS}_{2}$ score & 0.990 & $0.682-1.438$ & 0.959 & & & \\
\hline $\mathrm{CHADS}_{2}$ score $\geq 3$ & 3.560 & $1.589-7.978$ & 0.002 & 1.824 & $0.746-4.460$ & 0.188 \\
\hline $\mathrm{CHA}_{2} \mathrm{DS}_{2}$-VASc score & 1.003 & $0.772-1.302$ & 0.984 & & & \\
\hline $\mathrm{CHA}_{2} \mathrm{DS}_{2}$-VASc score $\geq 3$ & 3.952 & $1.878-8.315$ & $<0.001$ & 3.720 & $1.367-10.124$ & 0.010 \\
\hline $\mathrm{R}_{2} \mathrm{CHADS}_{2}$ score & 0.990 & $0.682-1.438$ & 0.959 & & & \\
\hline $\mathrm{R}_{2} \mathrm{CHADS}_{2}$ score $\geq 3$ & 1.998 & $0.693-5.755$ & 0.200 & & & \\
\hline Abnormal renal function & 1.004 & $0.479-2.104$ & 0.991 & & & \\
\hline Procedural termination & 1.001 & $0.482-2.078$ & 0.998 & & & \\
\hline
\end{tabular}

Abbreviations as in Table 1 .

${ }^{a}$ refers to total AF duration and duration of persistent AF prior to the initial procedure,

Procedural termination refers to atrial arrhythmias terminated and converted to sinus rhythm during ablation.

Table 5. Cox Proportional Hazard Analysis to Recognize Variables Associated with AF Recurrence after the Repeat Procedure.

\begin{tabular}{|c|c|c|c|c|c|c|}
\hline \multirow[t]{2}{*}{ Variables } & \multicolumn{3}{|c|}{ Univariate analysis } & \multicolumn{3}{|c|}{ Multivariate analysis } \\
\hline & HR & $95 \% \mathrm{CI}$ & $\mathrm{p}$ value & HR & $95 \% \mathrm{CI}$ & $\mathrm{p}$ value \\
\hline Age (years) & 0.986 & $0.952-1.021$ & 0.422 & & & \\
\hline Male & 0.610 & $0.292-1.272$ & 0.187 & & & \\
\hline Total AF duration (years) ${ }^{a}$ & 1.053 & $1.012-1.096$ & 0.011 & 1.019 & $0.979-1.062$ & 0.356 \\
\hline Duration of persistent AF (years) ${ }^{a}$ & 1.004 & $1.001-1.008$ & 0.011 & 1.001 & $0.997-1.005$ & 0.675 \\
\hline \multicolumn{7}{|l|}{ Echocardiographic parameters } \\
\hline LA diamension $(\mathrm{mm})$ & 1.007 & $0.939-1.080$ & 0.850 & & & \\
\hline $\operatorname{LVEF}(\%)$ & 1.052 & $0.953-1.160$ & 0.316 & & & \\
\hline \multicolumn{7}{|l|}{ Comorbidities (n) } \\
\hline Hypertension & 0.680 & $0.352-1.342$ & 0.272 & & & \\
\hline Diabetes mellitus & 1.178 & $0.457-3.039$ & 0.735 & & & \\
\hline $\mathrm{HCM}$ & 7.004 & $1.455-33.707$ & 0.015 & 3.080 & $0.584-16.250$ & 0.185 \\
\hline DCM & 1.753 & $0.240-12.560$ & 0.587 & & & \\
\hline COPD & 1.294 & $0.507-3.878$ & 0.660 & & & \\
\hline $\mathrm{CHADS}_{2}$ score & 0.827 & $0.575-1.190$ & 0.827 & & & \\
\hline $\mathrm{CHADS}_{2}$ score $\geq 3$ & 2.498 & $0.339-18.424$ & 0.369 & & & \\
\hline $\mathrm{CHA}_{2} \mathrm{DS}_{2}$-VASc score & 0.828 & $0.648-1.058$ & 0.132 & & & \\
\hline $\mathrm{CHA}_{2} \mathrm{DS}_{2}$-VASc score $\geq 3$ & 3.439 & $1.707-6.926$ & 0.001 & 2.707 & $1.151-6.363$ & 0.022 \\
\hline $\mathrm{R}_{2} \mathrm{CHADS}_{2}$ score & 0.843 & $0.617-1.152$ & 0.284 & & & \\
\hline $\mathrm{R}_{2} \mathrm{CHADS}_{2}$ score $\geq 3$ & 1.012 & $0.310-3.307$ & 0.984 & & & \\
\hline Abnormal renal function & 3.533 & $1.741-7.167$ & $<0.001$ & 2.821 & $1.277-6.232$ & 0.010 \\
\hline Procedural termination & 2.254 & $0.915-5.550$ & 0.043 & 2.048 & $0.810-5.230$ & 0.060 \\
\hline
\end{tabular}

Abbreviations as in Table 1.

${ }^{a}$ refers to total AF duration and duration of persistent AF prior to the initial procedure.

Procedural termination refers to atrial arrhythmias terminated and converted to sinus rhythm during the repeat ablation.

term follow-up.

dom from asymptomatic or symptomatic arrhythmia for pa-

Regarding the findings of the Kaplan-Meier analysis, free- tients with AT/AF recurrence was present in Fig. 3. 
A

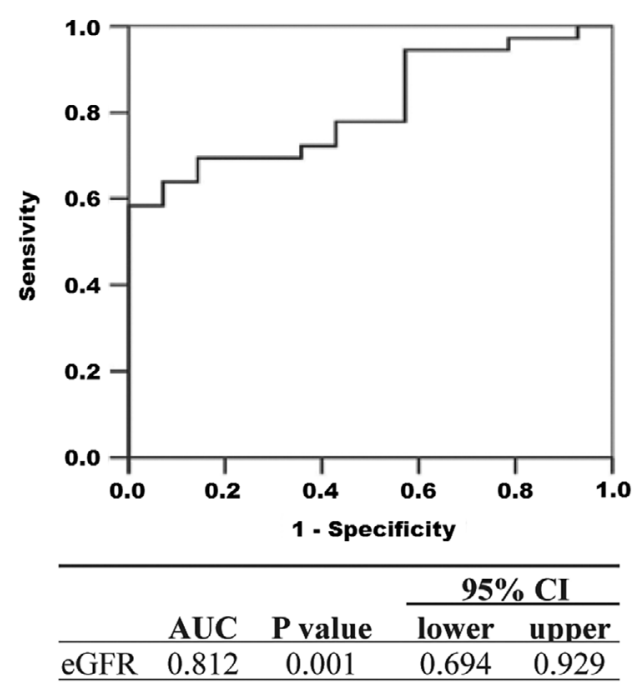

B

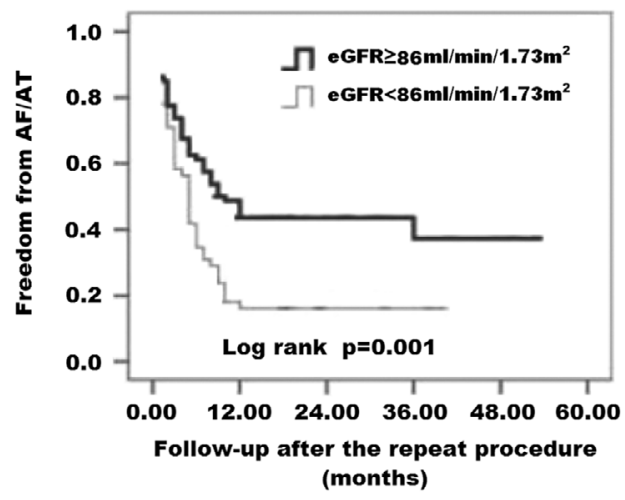

Figure 2. (A) Receiver operating curve of eGFR for an unsuccessful repeat catheter ablation procedure for patients with AF reccurrence. (B) Kaplan-Meier event-free survival curves after repeat catheter ablation for patients with $\mathrm{AF}$ recurrence. The optimal cut-off value of the eGFR in the prediction of AF recurrence was $86 \mathrm{~mL} / \mathrm{min} / 1.73 \mathrm{~m}^{2} . \log$ rank $\mathrm{p}=0.001$.

\section{Quality of life and EHRA class}

Because the period of follow-up was not identical for each patient, we consistently choose 24 months post reablation to judge the postoperative QOL and AF-related symptom classification. According to the follow-up results, we then divided enrolled patients into two groups named non-responders and responders. Non-responders are patients who demonstrated treatment failure for the repeat procedure and had recurrent atrial arrhythmia, while responders are patients who achieved and maintained SR after the redo procedure. As portrayed in Fig. 4, there was no significant difference between the responders and non-responders at QOL baseline. At 24 months post re-ablation, compared with baseline, all eight subscales of QOL showed a significant improvement in the patients maintaining SR (responder), while only two out of the eight subscales of QOL showed an improvement in the patients with recurrent atrial arrhythmias (non-responder). The change in proportion of each

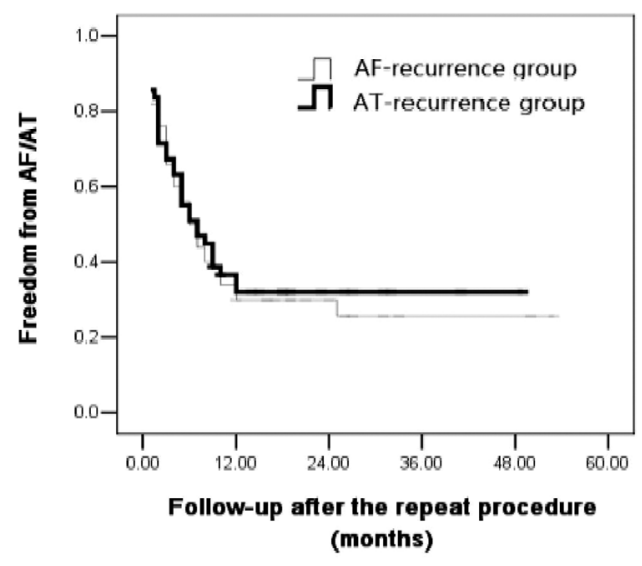

Numbers at risk

For AF recurrence

For AT recurrence

Total

$\begin{array}{rrrrr}50 & 15 & 6 & 2 & 0 \\ 49 & 16 & 7 & 2 & 1 \\ 99 & 31 & 13 & 4 & 1\end{array}$

Figure 3. Kaplan-Meier analysis of the long-term freedom from recurrences of atrial arrhythmias after the repeat catheter ablation for patients with AF recurrence and AT recurrence.

ERHA level demonstrated that maintaining SR after the repeat procedure successfully reduced the AF-related symptoms.

\section{Complications}

No atrial-esophageal fistula, significant PV stenosis and procedure-related deaths occurred. The complications were as follows: cardiac tamponade $(n=2$, both of the patients were treated conservatively with percutaneous pericardiocentesis), arteriovenous femoral fistulae $(\mathrm{n}=1)$, and femoral hematoma $(n=2)$.

\section{Discussion}

\section{Principal study findings}

We studied the outcome of a repeat procedure for longstanding persistent $\mathrm{AF}$ with an $\mathrm{AF}$ duration of more than 2 years and analyzed the predictors of recurrence after the repeat procedure. The main findings were as follows. (1) After a mean follow-up of 31 months, the overall success rates for redo AF ablation were low (30.3\%). Compared with AT recurrent patients, patients with $\mathrm{AF}$ recurrence had a lower success rate $(32.7 \%$ vs. $28.0 \%, \mathrm{p}=0.614)$. (2) $\mathrm{A} \mathrm{CHA}_{2} \mathrm{DS}_{2}-$ VASc score $\geq 3$ was a predictor of recurrence after the repeat procedure. (3) AF recurrent patients with eGFR $<86 \mathrm{~mL} /$ $\min / 1.73 \mathrm{~m}^{2}$ after undergoing the initial procedure of longstanding persistent $\mathrm{AF}$ were more prone to undergo a failed repeat ablation compared to those with eGFR $\geq 86 \mathrm{~mL} / \mathrm{min} /$ $1.73 \mathrm{~m}^{2}$. However, an abnormal renal function had no influ- 
A

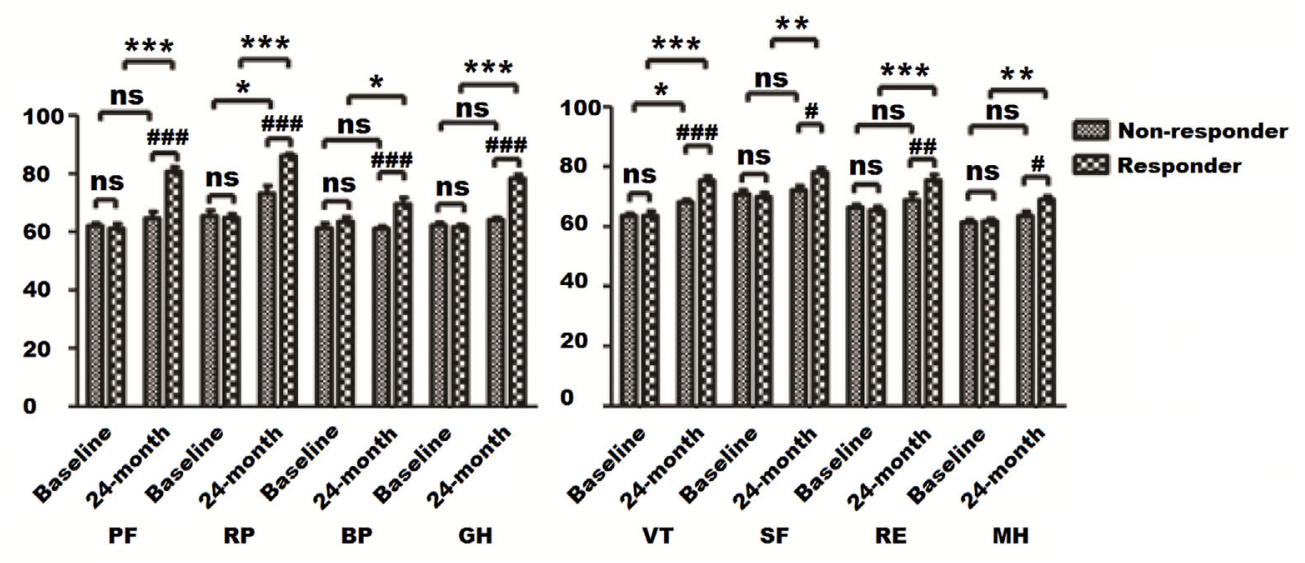

B
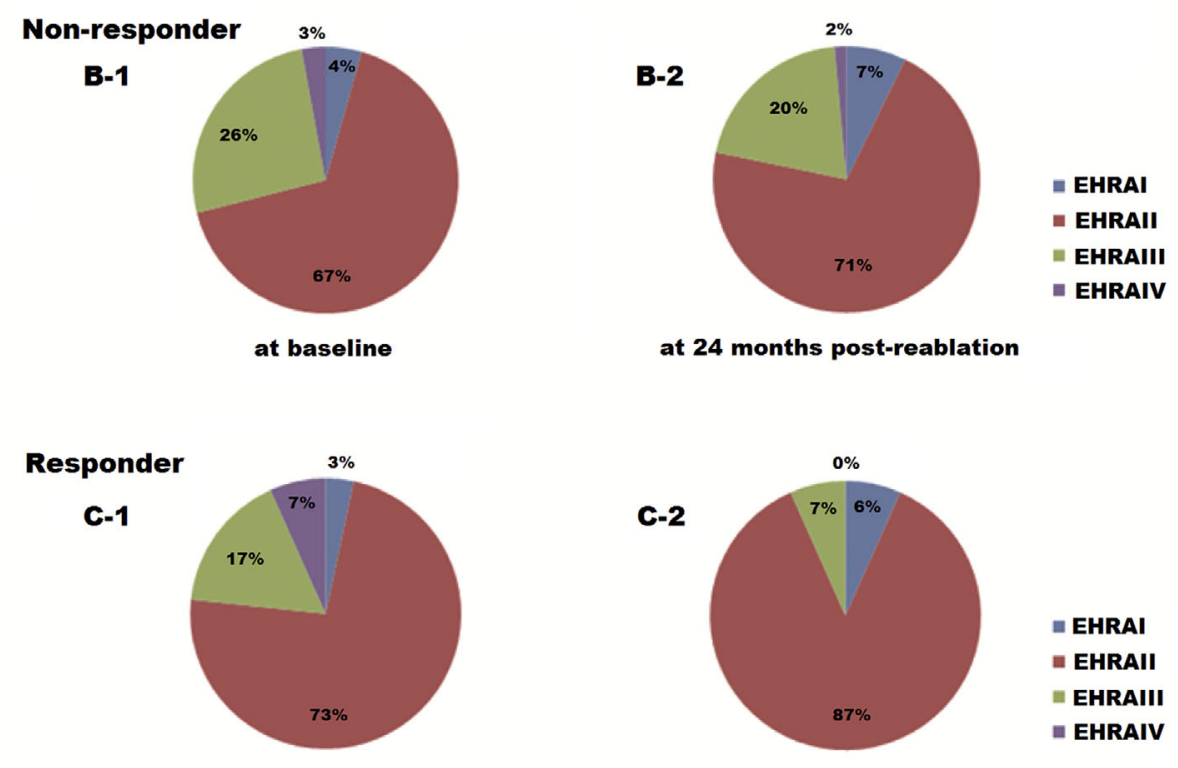

at baseline

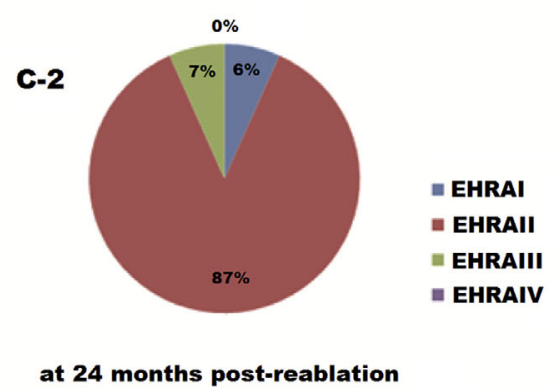

Figure 4. (A) SF-36 QOL component summaries for patients with sinus rhythm and those with recurrent atrial arrhythmias after the repeat procedure at baseline and at $\mathbf{2 4}$ months post-reablation. The range of scores for each subscales is $\mathbf{0}$ to 100 (with higher scores indicating better quality of life). ***p $<0.0001, * * \mathbf{p}<0.001, * \mathbf{p}<0.05$ vs. baseline; \#\#\#< $<0.0001, \# \# \mathbf{p}<0.001, \# \mathbf{p}<0.05$ vs. control. (B) The pie chart demonstrates the proportion of each EHRA level at baseline and at 24 months post-reablation for non-responders. (C) The pie chart demonstrates the proportion of each EHRA level at baseline and at 24 months post-reablation for responders. PF: physical functioning, RP: role physical, BP: body pain, GH: general health, VT: vitality, SF: social functioning, RE: role emotional, MH: mental health. Non-responder, patients failed in the repeat procedure and had recurrent atrial arrhythmias; Responder, patients restoring and maintaining sinus rhythm due to the repeat procedure.

ence on the outcome for AT recurrent patients. (4) In general, the QOL of the patients maintaining SR improved after the repeat procedure.

Long term results of repeat catheter ablation in long-standing persistent atrial fibrillation with an AF duration of more than 2 years

No studies have previously investigated the clinical predictors of re-ablation success in patients with long-standing persistent $\mathrm{AF}$ and a total $\mathrm{AF}$ duration of more than 2 years.
The majority of prior studies showed arrhythmia-free survival rates after single and multiple procedures. McCready et al. investigated 191 persistent AF patients who received catheter ablation from 2003 to 2009 and demonstrated the single procedure success rate to be $32.0 \%$ after a mean follow-up of $13.5 \pm 9.4$ months, while the overall success rate was $64.0 \%$ after a mean follow-up of $13.0 \pm 8.9$ months (4). Rostock et al. analyzed the outcome in 395 persistent AF patients and showed that with a single procedure, the recurrence rate was $73.0 \%$ (5). The recent study performed by 
Chao et al., which enrolled 88 long-standing persistent AF patients receiving a stepwise catheter ablation from 2006 to 2008 , noted a success rate of $28.4 \%, 47.7 \%$ and $51.1 \%$ after the first, second and multiple procedures, respectively (3). Further, Tilz et al. describes that during 5-year follow-up, single and multiple ablation procedure success was $20 \%$ and $45 \%$, respectively, for patients with long-standing persistent atrial fibrillation. For patients with a total AF duration of more than 2 years, the outcomes were unfavorable (6).

Considering the reasons for the difference between these outcomes, diverse populations, various types of ablation strategies, the definition of the recurrence, different types of recurrent arrhythmias after catheter ablation, and the accuracy of follow-up may together explain this discrepancy. In comparison with previous studies, we focused on the outcomes of patients with a total AF duration more than 2 years after the repeat procedure. Furthermore, all of the enrolled patients suffered from long-standing persistent AF, and they underwent the same ablation strategy in the initial procedure. During the repeat procedure, all patients reached current ablation endpoints, which meant pulmonary vein isolation, bidirectional block of lines, or the disappearance of CFAEs. The study excluded the effects of incomplete pulmonary vein isolation, insufficient ablation of CFAEs, and unblocked lines on the outcome of a repeat procedure. In addition, we pointed out that the recurrence rate after the repeat procedure for patients with long-standing persistent AF and a total AF duration more than 2 years was as high as $69.7 \%$, especially for patients with AF recurrence (72.0\%). Accordingly, due to the high recurrence rate, the decision to perform a repeat ablation for long-standing persistent AF should be made cautiously by physicians.

\section{$C H A_{2} D S_{2}-V A S c$ score and recurrence after catheter ablation during a long-term follow-up}

In the present study, we showed a $\mathrm{CHA}_{2} \mathrm{DS}_{2}$-VASc score $\geq 3$ to be associated with a poor outcome after the repeat procedure for patients with AF recurrence as well as AT recurrence. Recently, a series of studies have demonstrated the $\mathrm{CHA}_{2} \mathrm{DS}_{2}$-VASc score to be a significant predictor of adverse events in patients undergoing the catheter ablation (14). It also serves as a combined risk predictor of first cardiovascular hospitalization for patients with $\mathrm{AF}$ and a risk factor of recurrence after catheter ablation $(3,15,16)$. Furthermore, Park et al. proved that the electroanatomical remodeling of the left atrium (LA) was related to the $\mathrm{CHA}_{2} \mathrm{DS}_{2}$-VASc score in patients with non-valvular AF (17). Park and coworkers found the LA volume to be significantly higher in patients with high $\mathrm{CHA}_{2} \mathrm{DS}_{2}$-VASc score. A higher LA volume was closely related to a lower endocardial voltage of LA, which may help to classify the severity of the tissue pathology underlying AF before and after the catheter ablation procedure (18). The high $\mathrm{CHA}_{2} \mathrm{DS}_{2}$-VASc score, as a predictor of $\mathrm{AF}$ recurrence, implies that it does have something to do with a larger LA volume and a lower LA voltage, and plays a role in the electroanatomical remodel- ing of patients with AF (19).

\section{Estimated glomerular filtration rate and recurrence after catheter ablation during a long-term follow-up}

Recently published studies analyzed the association of an impaired renal function with post-ablation recurrence of AF (20-23). Chao et al. proved that even a mildly decreased eGFR was associated with an arrhythmogenic LA substrate and a high recurrence rate of catheter ablation in patients with paroxysmal AF (23). Tokuda et al. certified that low eGFR independently influenced the outcome of catheter ablation for paroxysmal AF (22). Our study found that for patients with $\mathrm{AF}$ recurrence prior to the repeat procedure, eGFR $<86 \mathrm{~mL} / \mathrm{min} / 1.73 \mathrm{~m}^{2}$ was a risk factor of recurrence for the redo procedure, which reconfirmed these results. Nevertheless, for patients with AT recurrence, an abnormal renal function did not help to predict the outcome of reablation.

Regarding the strong association between arrhythmia recurrence and a low eGFR after the repeat procedure, one possible explanation may be the correlation between an impaired renal function and established LA remodeling as already described (23). The rennin-angiotensin-aldosterone system (RAAS) plays a key role in the relationship between renal disease and cardiac disease. There is strong evidence that RAAS is involved in the genesis of AF (24). When the renal function is impaired, RAAS is activated, and the secretion of angiotensin II is known to increase. Further, angiotensin II increases the atrial pressure, promotes atrial fibrosis, and modulates ion channels (25). All of these effects are involved in atrial structural and electrical remodeling, thus triggering AF. Considering that the abnormal renal function has a different effect on the outcome of the patients with AF-recurrence and AT-recurrence, it is likely that for patients with AT recurrence prior to the repeat procedure, atrial structural and electrical remodeling were not so serious as for patients with AF recurrence prior to the repeat procedure.

In addition, emerging evidence suggests inflammation and oxidative stress play an important role in the pathophysiology of both AF and renal dysfunction (26). The proinflammatory and profibrotic state in the patients with moderate to severe renal impairment is more prone to result in a recurrence after the repeat procedure of AF. In brief, the patients with a low eGFR after the procedure were associated with advanced atrial remodeling, including structural and electrical changes, which were shown to result in recurrence after the repeat ablation (23).

\section{Quality of life and EHRA class}

Although AF is seldom immediately life threatening, it imposes a substantial QOL burden on many patients (27). Previous reports have demonstrated that for patients with persistent AF, the restoration and maintenance of SR after catheter ablation was associated with an improvement in QOL measures $(28,29)$. In our study, a significant improve- 
ment was observed in eight subscales of the SF-36 questionnaire for patients maintaining SR after the repeat procedure at 24 months post re-ablation; while only two out of the eight subscales of QOL improved in patients with recurrent atrial arrhythmias. The change in the proportion of each ERHA level indicated that maintaining SR after a repeat procedure could well reduce the AF-related symptoms. Improved QOL scores denoted the importance of the long-term maintenance of SR in patients with persistent AF.

\section{Study limitations}

There are several limitations associated with the present study. Firstly, this study was performed in a single-center with a relatively small sample size. Therefore, larger studies are required to confirm the validity of our observed results. Secondly, 11 patients (6.0\% of 182 recurrent patients) dropped out from the follow-up because they underwent ablation at other institutions. Exclusion of these patients may have influenced the results of this study. Thirdly, the routine follow-up investigations were limited by reliance on 12-lead surface electrocardiogram and 24-hour Holter monitoring. These assessments are less precise than continuous cardiac rhythm monitoring devices. It was likely that some asymptomatic recurrence of arrhythmias was undetected. This inevitably underestimated the recurrence rate. The patients who had undetected recurrence could thus have been included in the success group, perhaps some important predictors of the repeat procedure may be masked. Fourthly, renal disease and atrial fibrillation share common risk factors, including diabetes mellitus, hypertension and abnormal left ventricular systolic function, the extent to which eGFR is a direct causal link to AF or merely a marker of systemic disease is not clear. Finally, because the follow-up periods were not identical for each patient, the QOL was only assessed at baseline and at 24 months post re-ablation.

\section{Conclusion}

As a whole, the success rate of all patients with longstanding persistent $\mathrm{AF}$ and a total AF duration of more than 2 years undergoing repeat ablation is poor with the corollary that patients with a $\mathrm{CHA}_{2} \mathrm{DS}_{2}$-VASc score $\geq 3$. An impaired renal function in $\mathrm{AF}$ recurrent patients is a significant risk factor for a failed redo procedure. However, for those who do achieve durable SR, the QOL and AF symptomatology improve significantly.

The authors state that they have no Conflict of Interest (COI).

\section{Acknowledgement}

We are grateful to the participants, including the referring physicians and patients, for their help and cooperation during this study.

\section{Financial Support}

This work was supported in part by the grants from the Na- tional Natural Science Fund of China (81270161, 81370301, 81470372 and 81400244) and the Natural Science Fund of Shanghai, China (13ZR1438400 and 15ZR1438100).

\section{References}

1. Fuster V, Rydén LE, Cannom DS, et al. 2011 ACCF/AHA/HRS focused updates incorporated into the ACC/AHA/ESC 2006 Guidelines for the management of patients with atrial fibrillation: a report of the American College of Cardiology Foundation/ American Heart Association Task Force on Practice Guidelines developed in partnership with the European Society of Cardiology and in collaboration with the European Heart Rhythm Association and the Heart Rhythm Society. J Am Coll Cardiol 57: e101-e198, 2011.

2. Amerena JV, Walters TE, Mirzaee S, Kalman JM. Update on the management of atrial fibrillation. Med J Aust 199: 592-597, 2013.

3. Chao TF, Tsao HM, Lin YJ, et al. Clinical outcome of catheter ablation in patients with nonparoxysmal atrial fibrillation: results of 3-year follow-up. Circ Arrhythm Electrophysiol 5: 514-520, 2012.

4. McCready JW, Smedley T, Lambiase PD, et al. Predictors of recurrence following radiofrequency ablation for persistent atrial fibrillation. Europace 13: 355-361, 2011.

5. Rostock T, Salukhe TV, Steven D, et al. Long-term single- and multiple-procedure outcome and predictors of success after catheter ablation for persistent atrial fibrillation. Heart Rhythm 8: 13911397, 2011.

6. Tilz RR, Rillig A, Thum AM, et al. Catheter ablation of longstanding persistent atrial fibrillation: 5-year outcomes of the Hamburg Sequential Ablation Strategy. J Am Coll Cardiol 60: 19211929, 2012.

7. Weerasooriya R, Khairy P, Litalien J, et al. Catheter ablation for atrial fibrillation: are results maintained at 5 years of follow-up? J Am Coll Cardiol 57: 160-166, 2011.

8. Wójcik M, Berkowitsch A, Greiss H, et al. Repeated catheter ablation of atrial fibrillation: how to predict outcome? Circ J 77: 22712279, 2013.

9. Piccini JP, Stevens SR, Chang Y, et al. Renal dysfunction as a predictor of stroke and systemic embolism in patients with nonvalvular atrial fibrillation: validation of the $\mathrm{R}_{2} \mathrm{CHADS}_{2}$ index in the ROCKET AF (Rivaroxaban Once-daily, oral, direct factor Xa inhibition Compared with vitamin $\mathrm{K}$ antagonism for prevention of stroke and Embolism Trial in Atrial Fibrillation) and ATRIA (AnTicoagulation and Risk factors In Atrial fibrillation) study cohorts. Circulation 127: 224-232, 2013.

10. Levey AS, Coresh J, Balk E, et al. National Kidney Foundation practice guidelines for chronic kidney disease: evaluation, classification, and stratification. Ann Intern Med 139: 137-147, 2003.

11. Liu X, Tan HW, Wang XH, et al. Efficacy of catheter ablation and surgical CryoMaze procedure in patients with long-lasting persistent atrial fibrillation and rheumatic heart disease: a randomized trial. Eur Heart J 31: 2633-2641, 2010.

12. Dorian P, Guerra PG, Kerr CR, et al. Validation of a new simple scale to measure symptoms in atrial fibrillation: the Canadian Cardiovascular Society Severity in Atrial Fibrillation scale. Circ Arrhythm Electrophysiol 2: 218-224, 2009.

13. Kirchhof $\mathrm{P}$, Auricchio A, Bax J, et al. Outcome parameters for trials in atrial fibrillation: executive summary. Eur Heart J 28: 28032817, 2007.

14. Chao TF, Lin YJ, Tsao HM, et al. CHADS 2 and $\mathrm{CHA}_{2} \mathrm{DS}_{2}-\mathrm{VASc}$ scores in the prediction of clinical outcomes in patients with atrial fibrillation after catheter ablation. J Am Coll Cardiol 58: 23802385, 2011.

15. Naccarelli GV, Panaccio MP, Cummins G, Tu N. CHADS 2 and $\mathrm{CHA}_{2} \mathrm{DS}_{2}$-VASc risk factors to predict first cardiovascular hospitalization among atrial fibrillation/atrial flutter patients. Am J Car- 
diol 109: 1526-1533, 2012.

16. Kornej J, Hindricks G, Kosiuk J, et al. Comparison of $\mathrm{CHADS}_{2}$, $\mathrm{R}_{2} \mathrm{CHADS}_{2}$, and $\mathrm{CHA}_{2} \mathrm{DS}_{2}-\mathrm{VASc}$ scores for the prediction of rhythm outcomes after catheter ablation of atrial fibrillation: the Leipzig Heart Center AF Ablation Registry. Circ Arrhythm Electrophysiol 7: 281-287, 2014.

17. Park JH, Joung B, Son NH, et al. The electroanatomical remodelling of the left atrium is related to $\mathrm{CHADS}_{2} / \mathrm{CHA}_{2} \mathrm{DS}_{2} \mathrm{VASc}$ score and events of stroke in patients with atrial fibrillation. Europace 13: 1541-1549, 2011.

18. Verma A, Wazni OM, Marrouche NF, et al. Pre-existent left atrial scarring in patients undergoing pulmonary vein antrum isolation: an independent predictor of procedural failure. J Am Coll Cardiol 45: 285-292, 2005.

19. Park JH, Pak HN, Choi EJ, et al. The relationship between endocardial voltage and regional volume in electroanatomical remodeled left atria in patients with atrial fibrillation: comparison of three-dimensional computed tomographic images and voltage mapping. J Cardiovasc Electrophysiol 20: 1349-1356, 2009.

20. Berkowitsch A, Kuniss M, Greiss H, et al. Impact of impaired renal function and metabolic syndrome on the recurrence of atrial fibrillation after catheter ablation: a long-term follow-up. Pacing Clin Electrophysiol 35: 532-543, 2012.

21. Naruse $Y$, Tada H, Sekiguchi Y, et al. Concomitant chronic kidney disease increases the recurrence of atrial fibrillation after catheter ablation of atrial fibrillation: a mid-term follow-up. Heart Rhythm 8: 335-341, 2011.

22. Tokuda M, Yamane T, Matsuo S, et al. Relationship between renal function and the risk of recurrent atrial fibrillation following catheter ablation. Heart 97: 137-142, 2011.

23. Chao TF, Lin YJ, Chang SL, et al. Associations between renal function, atrial substrate properties and outcome of catheter ablation in patients with paroxysmal atrial fibrillation. Circ J 75: 23262332, 2011.

24. Iravanian S, Dudley SC Jr. The renin-angiotensin-aldosterone system (RAAS) and cardiac arrhythmias. Heart Rhythm 5: S12-S17, 2008.

25. Goette A, Staack T, Röcken C, et al. Increased expression of extracellular signal-regulated kinase and angiotensin-converting enzyme in human atria during atrial fibrillation. J Am Coll Cardiol 35: 1669-1677, 2000.

26. Li J, Solus J, Chen Q, et al. Role of inflammation and oxidative stress in atrial fibrillation. Heart Rhythm 7: 438-444, 2010.

27. Thrall G, Lane D, Carroll D, Lip GY. Quality of life in patients with atrial fibrillation: a systematic review. Am J Med 119: 448.e1-448.e19, 2006.

28. Singh SN, Tang XC, Singh BN, et al; SAFE-T Investigators. Quality of life and exercise performance in patients in sinus rhythm versus persistent atrial fibrillation: a Veterans Affairs Cooperative Studies Program Substudy. J Am Coll Cardiol 48: 721730, 2006.

29. Reynolds MR, Walczak J, White SA, Cohen DJ, Wilber DJ. Improvements in symptoms and quality of life in patients with paroxysmal atrial fibrillation treated with radiofrequency catheter ablation versus antiarrhythmic drugs. Circ Cardiovasc Qual Outcomes 3: 615-623, 2010.

The Internal Medicine is an Open Access article distributed under the Creative Commons Attribution-NonCommercial-NoDerivatives 4.0 International License. To view the details of this license, please visit (https://creativecommons.org/licenses/ by-nc-nd/4.0/).

(C) 2016 The Japanese Society of Internal Medicine http://www.naika.or.jp/imonline/index.html 